\title{
Visualization of Flow inside a Regenerative Turbomachinery
}

\author{
Yang Hyeonmo $^{1}$, Lee Kyoung-yong ${ }^{1}$, Choi Youngseok ${ }^{1}$ and Jeong Kyungseok ${ }^{2}$ \\ ${ }^{1}$ Thermal \& Fluid Systems R\&BD Group, Korea Institute of Industrial Technology, \\ 89, Yangdaegiro-gil, Ipjang-myeon, Seobuk-gu, Cheonan-si, Chungcheongnam-do, 331-822, KOREA, \\ yhm0430@kitech.re.kr, chrisst@kitech.re.kr, yschoi@kitech.re.kr \\ ${ }^{2}$ Dept. of Mechanical Engineering, Korea University of Technology and Education, \\ 1600, Chungjeol-ro, Byeongcheon-myeon, Dongnam-gu, Cheonan-si, Chungcheongnam-do, 330-861, \\ KOREA, jks@koreatech.ac.kr
}

\begin{abstract}
In this study, we visualized the internal flow of a regenerative turbomachinery using the direct injection tracer method. For visualization, the working fluid was water and the tracer was oil colors (marbling colors). Droplets were injected at the inlet of the machinery and the streak were recorded using a high-speed camera with high-power light sources. While circulating inside the groove, the droplets were translated by the rotational motion of the impeller. When the droplets flow out of the impeller groove, relative to the impeller, they moved more slowly. And the droplets repeatedly reentered into the groove and circulated again. Then the droplets either flowed to the outlet or to the stripper. As a result, this experiment has confirmed the internal circulating flow of a regenerative turbomachinery.
\end{abstract}

Keywords: Regenerative Turbomachinery, Internal Circulation Flow, Flow Visualization, Direct Injection Tracer Method.

\section{Introduction}

For achieving high pressure and low flow rate, regenerative turbomachinery is the preferred method, as it can achieve the highest efficiency at a low specific speed. Usually, it is used as the fuel pump of a vehicle fuel delivery system, domestic water pump, or industrial high pressure blower. Recently, research has been ongoing to apply such devices to supply air to a stack of domestic fuel cells or to hydrogen recirculation devices.

The principle of regenerative turbomachinery is founded on the use of an impeller which is equipped with vanes. This rotates radially in the case and the water head rises by momentum exchange of the fluid. In this process, the fluid assumes a circulatory flow between the impeller and channel. The circulatory flow has been shown by the result of Choi [1] and Shim [2] using numerical experiments; for this study, however, we were unable to find their experimental study.

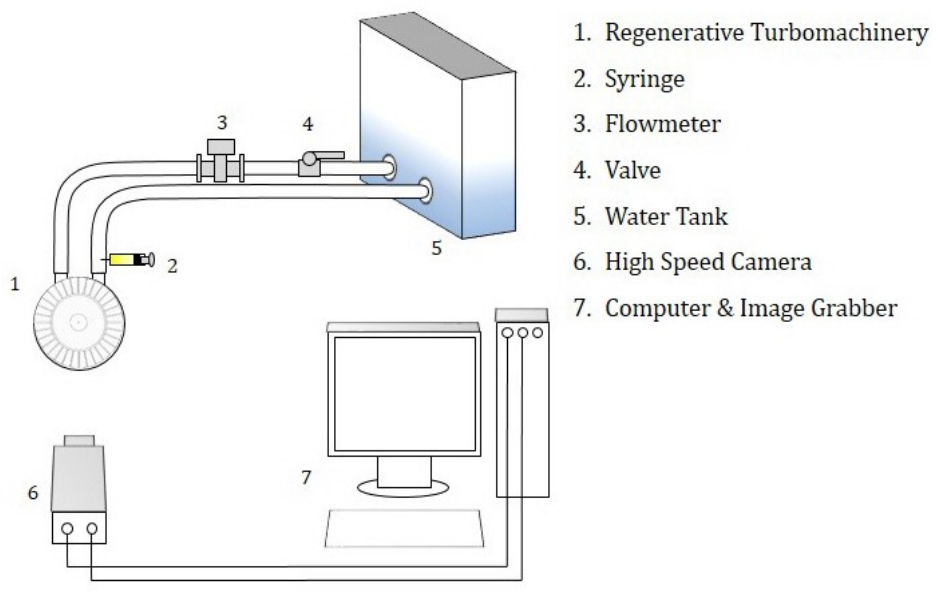

Fig. 1 Schematic arrangement of flow visualization 


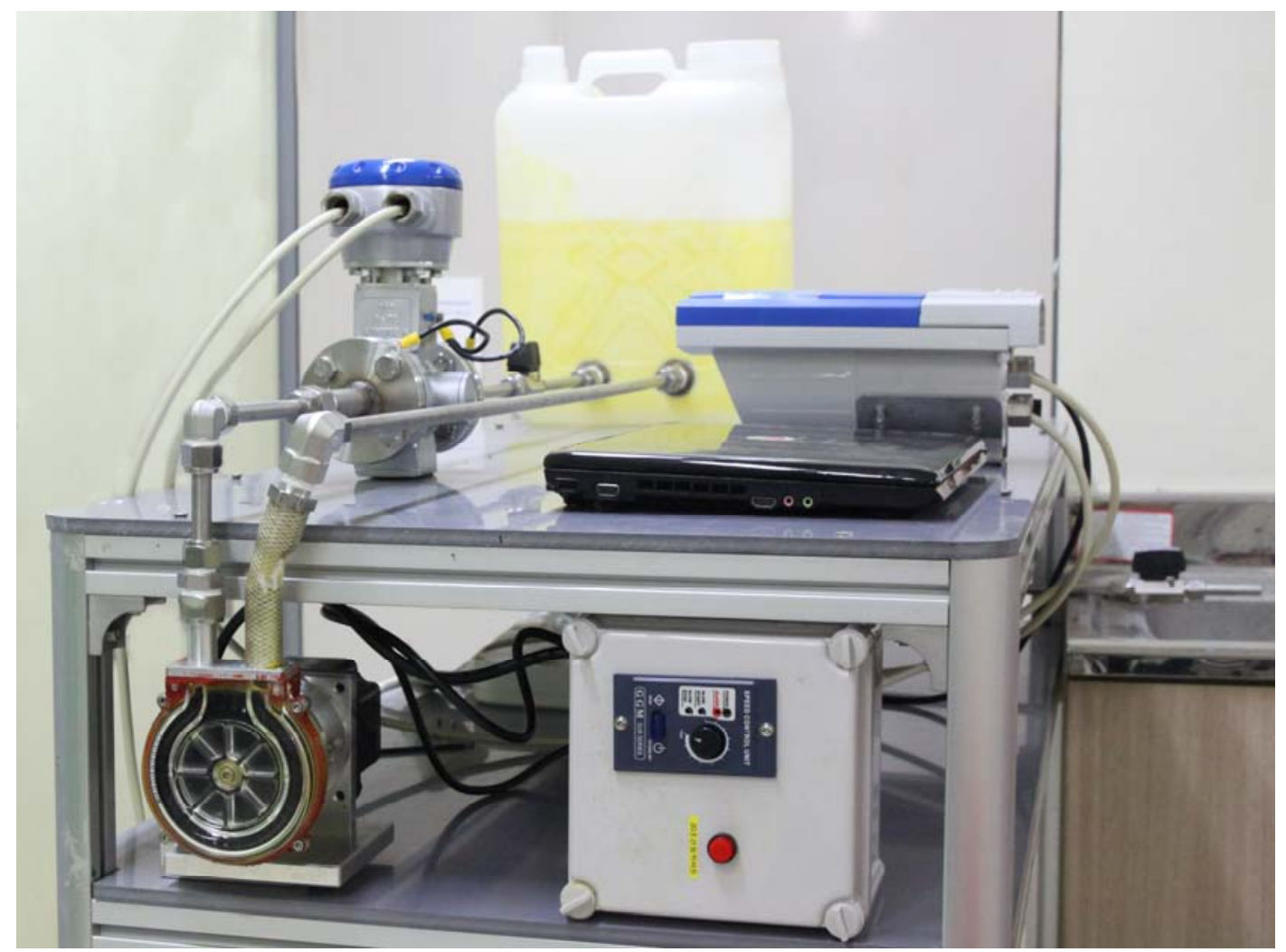

Fig. 2 View of experimental equipment

In this study, flow visualization using the direct injection tracer method was performed to examine the inner circulatory flow of side-channel regenerative turbomachinery.

\section{Experiment}

Figure 1 shows a schematic arrangement depicting the flow visualization. The regenerative blower used in the experiment is the side channel type; it blows air into the fuel cell. For visualization, the working fluid has been changed to water, and the blower works like a pump. The case was made of acrylic and the impeller was colored black. The direction of inlet and outlet is upwards to prevent the generation of bubbles. Inlet and outlet are connected with a water tank to create a closed loop. The droplets are injected by syringe near the inlet, and the flow rate is measured by a magnetic flowmeter. The experiment was set up as shown in Figure 2.

When the working fluid is air, the rated revolutions per minute of the impeller are $6000 \mathrm{rpm}$. In this experiment, as the working fluid was water, the rotation velocity should be changed to about $400 \mathrm{rpm}$, which is the same as the Reynolds number. In this experiment, the rotation velocity of impeller was $377.36 \mathrm{rpm}$, and the flow rate was 6.75 lpm. The rotation direction and inlet and outlet are shown in Figure 3.

The droplets were oil colors, which are known as marbling colors. Although the density of the droplets was lower than water, the droplets were not mixed with the water and had high cohesiveness. For this reason, we decided that marbling colors could be used for the visualization. Before the experiment, we confirmed by repeated test that the droplets moved by inner flow, and did not float due to density difference.

The experiment was recorded by high-speed camera that takes 1000 frames per second with 1/8000 sec and 512 $\times 512$ pixels.

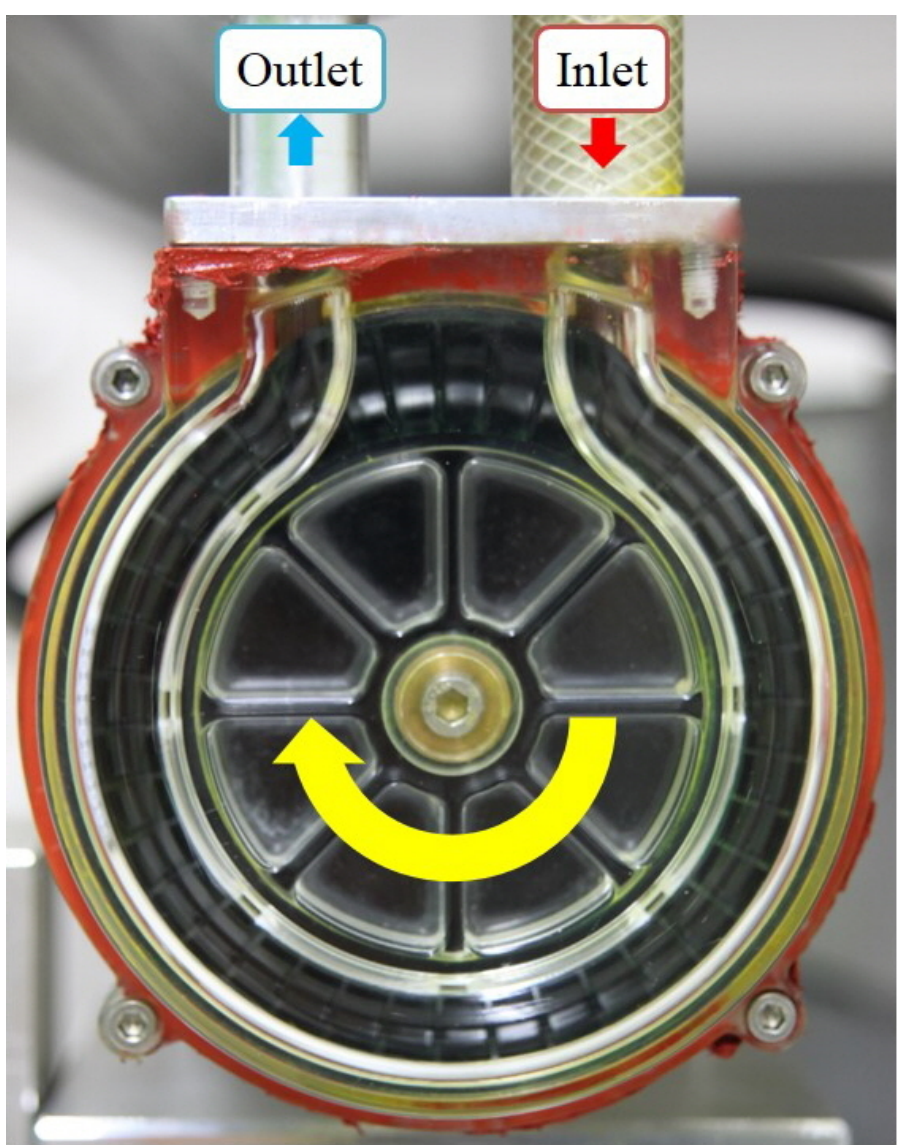

Fig. 3 Rotation direction and direction of inlet and outlet 


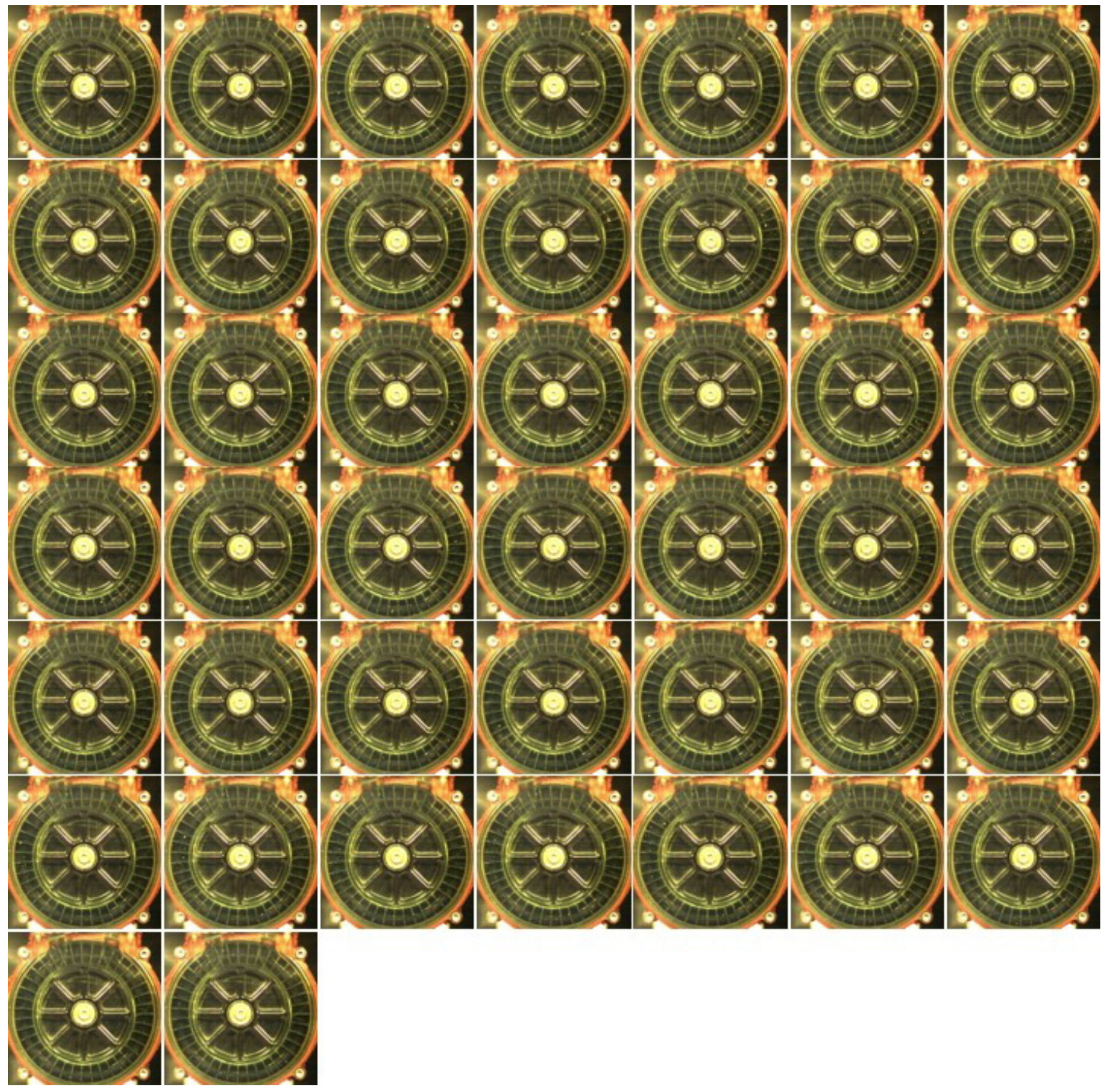

Fig. 4 Photography results

\section{Results}

Figure 4 shows the photography results. Among these result pictures, we have chosen pictures that are overlapping. Subsequently, we cropped the droplets position in each picture and added these as Figure 5. Figure 5 shows the internal circulation flow, which looks like a helical flow pattern when viewed from the front of the impeller. When the impeller rotated one time, the droplets showed a circulation flow for 3 to 5 times.

In these results, the circulation flow can be explained by two regions as is described in the following.

\subsection{Impeller region}

The fluid is drawn into the minimum radius section of the impeller groove. Figure 6 shows the movement path of the droplets in the impeller groove region. The fluid first enters varying sections of the impeller groove, but the movements are similar in that the droplets move from the minimum radius section to the maximum radius section of the impeller along the impeller groove and move clockwise along the impeller rotation.

Subsequently, the droplets that have finished moving in the channel reenter into the impeller groove and repeat the process again. About 3 to 4 times per one revolution of the impeller, the droplets are locked in and moved in the impeller groove.

Because the impeller rotation speed is higher than the flow rate in the channel, the fluid in the impeller groove is discharged to the channel with a higher speed relative to the channel flow. 


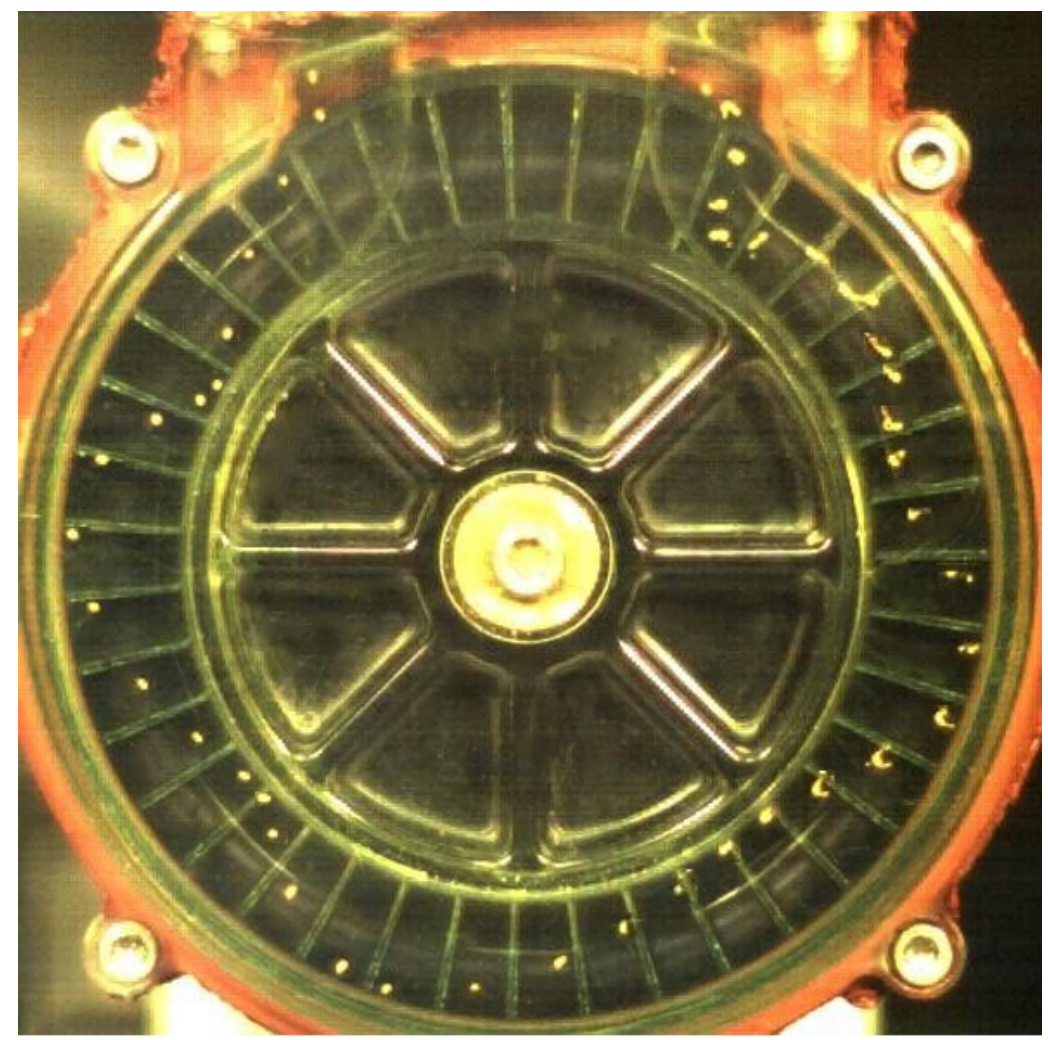

Fig. 5 Internal circulation flow of regenerative turbomachinery

In the case of the droplets that have been locked in the impeller groove while passing by the stripper and are moving to the inlet region, these move to the maximum radius section of the impeller groove from where the impeller meets the channel, as shown in Figure 7, and then are discharged to the channel.

Subsequently, the same as before, the droplets reenter to the impeller groove and the process is repeated 2 to 3 more times.

\subsection{Channel region}

When the fluid that has been locked in the impeller groove is moved by the rotation of the impeller, the resulting energy discharges the fluid to the channel. The fluid is moved to the center of the impeller along the cross-section shape of the channel, while being moved along the main flow rate of the channel at the same time.

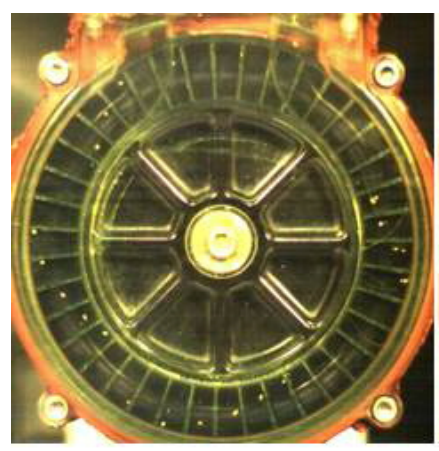

(a)

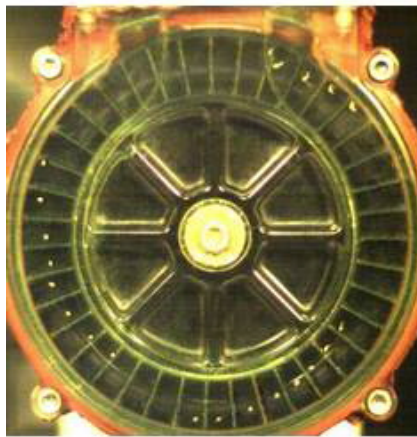

(d)

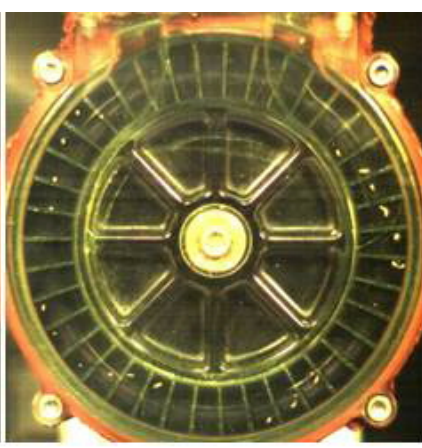

(b)

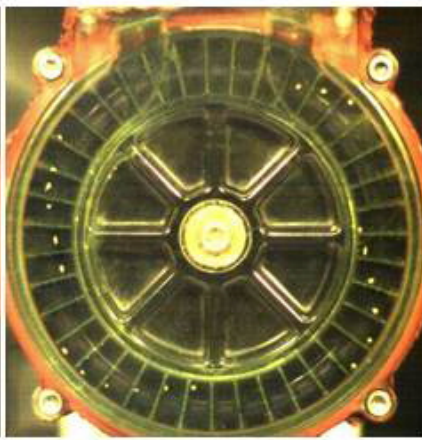

(e)

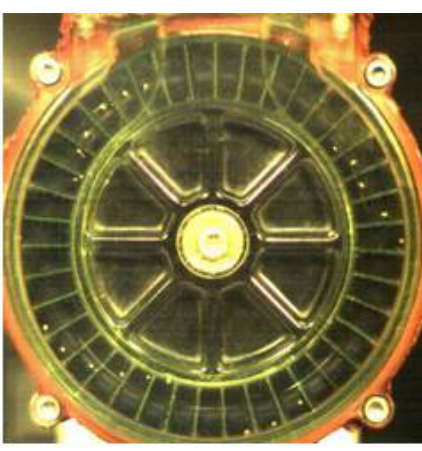

(c)

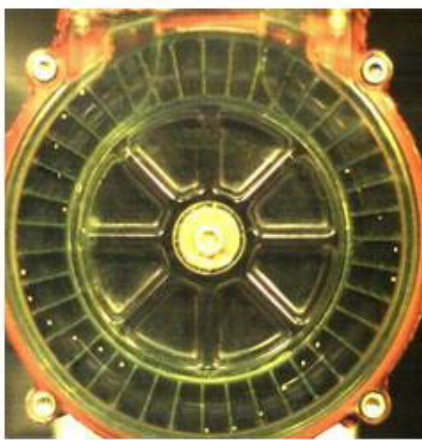

(f)

Fig. 6 Flow field of the impeller region from inlet 


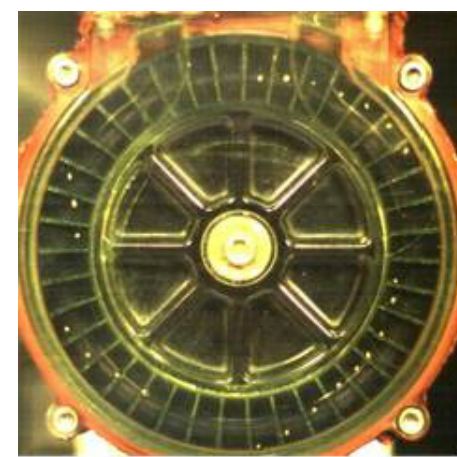

(a)

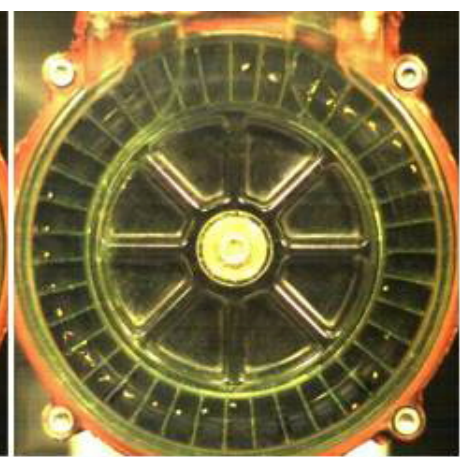

(b)

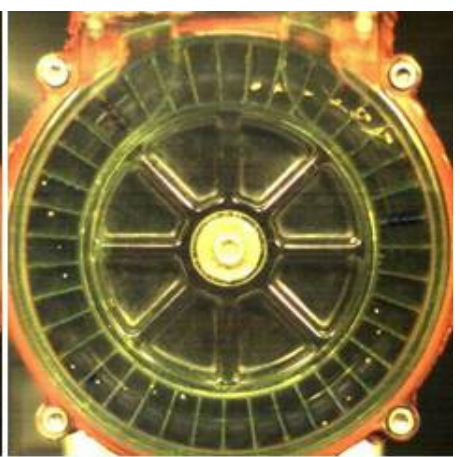

(c)

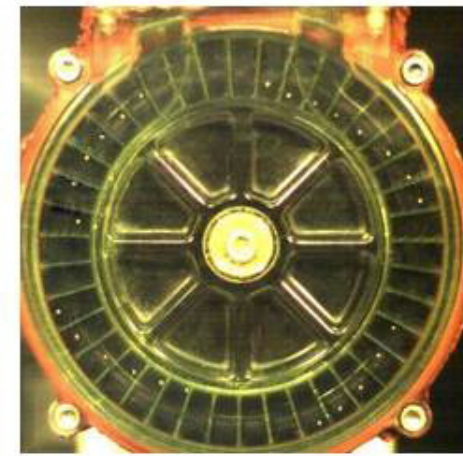

(d)

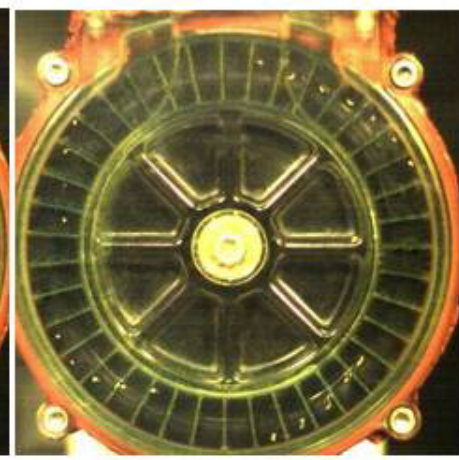

(e)

Fig. 7 Flow field of the impeller region from the stripper

Here, the droplets move a shorter distance and more slowly than was the case while in the impeller groove. Next, the droplets flow into the minimum radius section of the impeller groove. At this time, the droplets move more slowly than the rotation speed of the impeller, reenter only partially into the impeller groove that is discharging, and for the most part enter into the next impeller groove. Figure 8 shows the moving path of the droplets in the channel region.

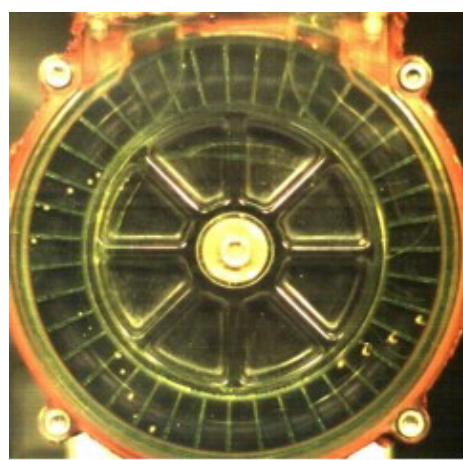

(a)

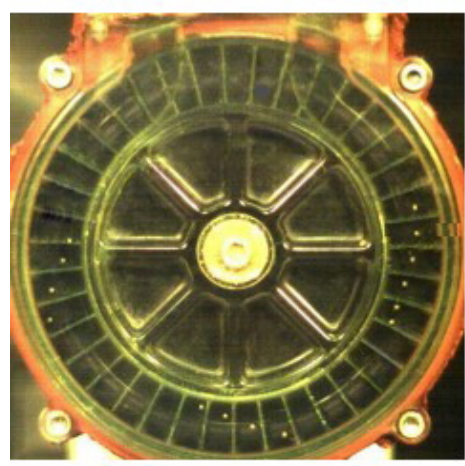

(d)

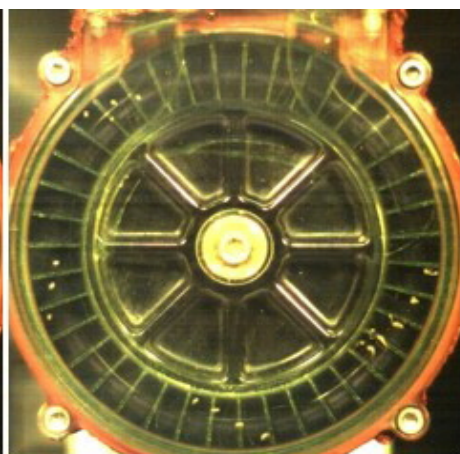

(b)

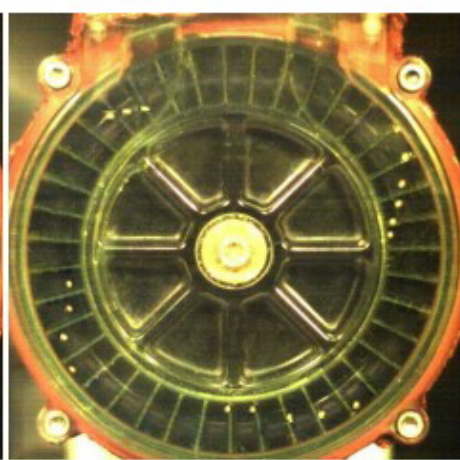

(e)

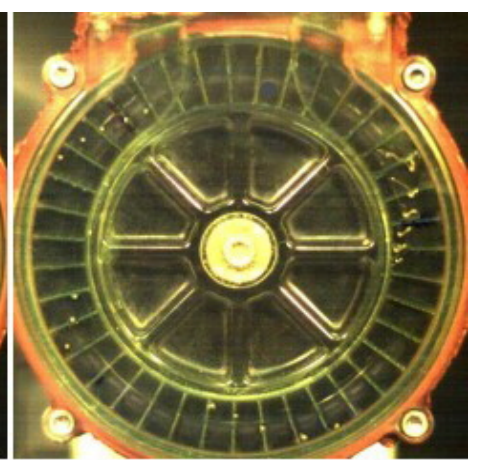

(c)

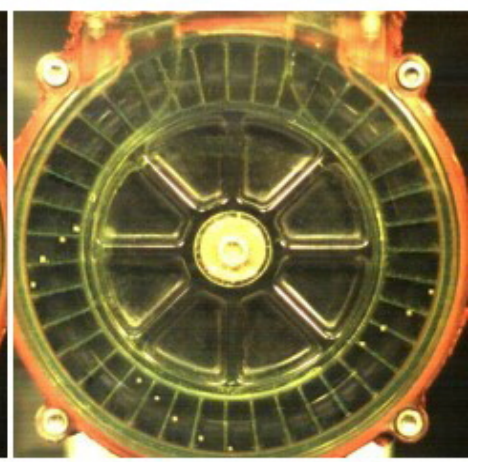

(f)

Fig. 8 Flow field of the channel region 


\section{Conclusion}

In this experiment, the direct injection tracer method was used to visualize the internal flow of regenerative turbomachinery. The internal circulating flow between the impeller and the case of the regenerative turbomachinery was investigated in an experiment. The internal circulation flow is as in the following:

(1) The internal circulation flow can be divided between the impeller region and the channel region.

(2) The fluid is discharged to the channel after flowing into the impeller groove.

(3) After being discharged to the channel, the fluid moves along the main stream of the channel.

(4) The fluid reenters to the impeller groove and repeats the flow circulation several times.

(5) Finally, the fluid is discharged from the outlet.

\section{Acknowledgments}

This research was supported by the Korea Institute of Industrial Technology Evaluation and Planning (ITEP) grant funded by the Ministry of Knowledge Economy (No. 10031962)

\section{References}

[1] Choi, Y. S. and Lee, K. Y., 2005, “Introduction to Development Challenges of Regenerative Turbomachinery,” Journal of Fluid Machinery, Vol. 8, No. 6, pp. 90-94.

[2] Shim, C. Y., Hong, C. O., and Kim, Y. S., 2005, "Development of Hydrogen Recirculation Blower for Fuel Cell Vehicle by Flow Analysis,” Proceedings of the KRMA Annual Meeting 2005, pp. 684-689. 